\title{
MicroRNA-146a as a potential regulator involved in the pathogenesis of atopic dermatitis
}

\author{
FENGGEN YAN $^{1 *}$, WEIWEI MENG ${ }^{2 *}$, SIQI YE ${ }^{1}$, XIAN ZHANG $^{1}$, XIUMEI MO ${ }^{1}$, \\ JUNFENG LIU ${ }^{1}$, DACAN CHEN ${ }^{1}$ and YING LIN ${ }^{1}$ \\ ${ }^{1}$ Department of Dermatology, The Second Affiliated Hospital of Guangzhou University of Chinese Medicine, \\ Guangdong Provincial Hospital of Chinese Medicine, Guangzhou, Guangdong 510120; \\ ${ }^{2}$ Department of Dermatology, The Second Affiliated Hospital of Henan University of Chinese Medicine, \\ Henan Provincial Hospital of Chinese Medicine, Henan, Zhengzhou 450000, P.R. China
}

Received December 17, 2018; Accepted August 23, 2019

DOI: $10.3892 / \mathrm{mmr} .2019 .10695$

\begin{abstract}
Previous studies have demonstrated that microRNA (miR)-146a is involved in the inflammatory response of atopic dermatitis (AD). The aim of the present study was to investigate the expression of miR-146a in the serum of patients with $\mathrm{AD}$ and in skin lesions of $\mathrm{AD}$ animal models. In addition, we aimed to predict and verify the target genes of miR-146a. miR-146a expression was measured in AD patient serum via reverse transcription-quantitative PCR. T-helper (Th) 1 [CD4 $4^{+}$; interferon (IFN)- $\left.\gamma^{+}\right]$and Th2 $\left[\mathrm{CD}^{+}\right.$; interleukin (IL) $\left.-4^{+}\right]$expression in peripheral blood mononuclear cells was evaluated using flow cytometry. Following the establishment of a 2,4-dinitrofluorobenzene-induced C57BL/6 mouse AD model, Th1 $\left(\mathrm{CD} 4{ }^{+} \mathrm{IFN}-\gamma^{+}\right)$and Th2 (CD4 $\left.4^{+} \mathrm{IL}-4^{+}\right)$expression was analyzed in murine spleen cells via flow cytometry. Plasmids were transfected into $293 \mathrm{~T}$ cells and at $48 \mathrm{~h}$ post-transfection, cells were analyzed using a luciferase assay system. The results revealed that the $\mathrm{AD}$ group had a significantly lower Th1/Th2 ratio and a significantly higher miR-146a expression compared with the control group $(\mathrm{P}<0.05)$. Furthermore, a decreased Th1/Th2 ratio and a significantly increased miR-146a expression were observed in the model group compared with the control group $(\mathrm{P}<0.01)$. We also conducted a dual-luciferase assay to determine whether small ubiquitin-related modifier 1 (SUMO1) if the target gene of miR-146a. We observed a $\sim 30 \%$ decrease in the relative luciferase activity in cells containing the 3 '-untranslated region of SUMO1 + miR-146a). The results of the luciferase assay indicated
\end{abstract}

Correspondence to: Professor Dacan Chen, Department of Dermatology, The Second Affiliated Hospital of Guangzhou University of Chinese Medicine, Guangdong Provincial Hospital of Chinese Medicine, 111 Dade Road, Guangzhou, Guangdong 510120, P.R. China

E-mail: cdc@gzucm.edu.cn

${ }^{*}$ Contributed equally

Key words: microRNA-146a, atopic dermatitis that may be a direct mRNA target of miR-146a; however, the quantification of band density of SUMO1 expression following western blotting did not significantly differ. The development of animal models in AD research is of vital importance. The results revealed that miR-146a may be a potential regulator involved in the pathogenesis of AD. Furthermore, the current study determined that miR-146a could be a valuable marker of AD and thus, may be applied in the development of therapeutic strategies for treating AD.

\section{Introduction}

Atopic dermatitis (AD) is a chronic, relapsing inflammatory skin disease that commonly occurs in children and teenagers. It is characterized by recurrent severe itching and eczema lesions in an atopic population (1). The morbidity of $\mathrm{AD}$ is increasing annually, particularly in developed countries and regions of rapid urbanization, and affects $15-30 \%$ of children and $2-10 \%$ of adults worldwide (2). This trend has also been observed in China; the AD morbidity rate of children aged 3-6 years was 8.3\% in Shanghai in 2012 (3) and 12.94\% in 1-7 year-olds in China in 2016 (4). The burden and recurrence of AD greatly reduces patient quality of life and places a significant economic burden on their households, creating potentially severe social impacts. The exact cause of AD is unclear, but it may result from a combination of genetic predispositions and environmental factors. Currently, it has been considered that a predominant T-helper (Th)2 immune response arising from an imbalance of Th1/Th2 cells (the two major types of CD4+ T helper cell) is a classical pathway of the disease (5).

MicroRNAs (miRNA/miR) are a type of non-coding small RNA molecule that is 21-25 nucleotides in length. They are involved the post-transcriptional regulation of endogenous gene expression by binding to specific mRNA sequences via base pairing, resulting in the subsequent recruitment of a silencing complex that degrades target mRNA or prohibit its translation $(6,7)$. Such regulation can lead to abnormalities in protein synthesis $(8,9)$, which may be associated with various human diseases $(10,11)$. miR-146a is a known anti-inflammatory and NF- $\mathrm{B}$ pathway-dependent miRNA that is expressed in a variety of immune cells, including B cells, T cells, monocytes and dendritic cells (12-15). It serves as 
a crucial regulatory factor of innate and adaptive immunity, and controls immune cell differentiation, antibody production and inflammatory factor secretion (16). A previous study revealed an increased miR-146a expression in the keratin and lesions of skin in patients with $\mathrm{AD}(17)$; however, its expression status in patient serum remains unclear.

The aim of the present study was to determine the expression of miR-146a in the serum of patients with AD and to establish a 2,4-dinitrofluorobenzene (DNFB)-induced AD-like skin lesion mouse model to investigate whether an imbalance in the Th1/Th2 ratio of murine spleen cells is consistent with that demonstrated in patients with AD. The present study also aimed to establish the expression status of miR-146a in skin lesions in a mouse model.

\section{Materials and methods}

Ethics statement. The use of human samples was approved by the Ethics Committee of Guangdong Provincial Hospital of Chinese Medicine (approval no. B2015-017-01). Written informed consent was obtained from each participant prior to enrollment.

Patients. A total of 25 patients with moderate-to-severe AD (19 males, 6 females; age range, 5-33 years; mean age 17.8 years; disease span range, 1-22 years; average length of disease, 9.64 years) participated in the current study. All patients were eligible for assessment using the Hanifin and Rajka criteria (18) (5 years old or older with a disease span $>1$ year), and Investigator Global Assessment criteria (scores 23) (19). No patients had received systematic hormone or immunosuppressor treatment within 6 months, or partial treatment of hormones and calcineurin inhibitors within 2 weeks prior to study commencement. No patients exhibited any psychological, tumor, cardiovascular, liver, kidney, brain or hematopoietic system disorders. The healthy control group comprised 16 individuals ( 9 males, 7 females; age range, 10-35 years; average age, 18.2 years). No individual in the control group possessed the aforementioned diseases or AD, asthma, allergic rhinitis or other allergic disorders.

Animals. Specific pathogen-free (SPF) C57BL/6 male mice (age, 6-8 weeks; weight, 16-22 g) were purchased from Guangdong Medical Science Experiment Center (batch no. 44007200026387). Mice were raised in an SPF grade animal room in the animal center of Guangdong Traditional Chinese Medicine Academy of Sciences. Animal care and experiments were conducted in accordance with the Laboratory Animal Research Ethical Committee Guidelines of Guangdong Provincial Hospital of Chinese Medicine, and was approved by the Laboratory Animal Ethics Committee of Guangdong Provincial Hospital of Chinese Medicine, approval no. 2015016). The room temperature was maintained at $25 \pm 2^{\circ} \mathrm{C}$ with $50-60 \%$ relative humidity. The circadian rhythm was set to $12 \mathrm{~h}$, and food and water were supplied freely. All experiments followed national regulations (Guangdong Provincial Hospital of Chinese Medicine, Guangdong Provincial Academy of Chinese Medical Sciences, Public Laboratory Animal Experiment Center, Standard Operating Procedure $\mathrm{PH}-\mathrm{C032}$ to $\mathrm{PH}-\mathrm{C036}$ ) on the usage, welfare and ethics of experimental animals.
Reagents. Human peripheral blood lymphocyte separation liquid (Tianjin Haoyang Biological), the serum miRNA extraction kit (Qiagen $\mathrm{GmbH}$ ), 2,4-dinitrofluorobenzene (DNFB, Sigma-Aldrich; Merck KGaA), the human and mouse Th1/Th2 cytokine kit (BD Biosciences), TRIzol ${ }^{\circledR}$ reagent (Thermo Fisher Scientific, Inc.), reverse transcription kit (Thermo Fisher Scientific, Inc.), real-time qPCR kit (Roche Diagnostics), miR-146a primer and miR-39 primer (Guangzhou Ruibo Biological Technology, Co., Ltd.) were used in the following experiments. The 3'-untranslated region (UTR) of small ubiquitin-related modifier 1 (SUMO1) luciferase vectors (GV272/SUMO1 3'-UTR wild type (wt) and GV272/SUMO1 3'-UTR mutant (mu)] was synthesized and cloned into GV272 (SV40-Luc-MCS, Fig. S1) firefly luciferase reporter vector by Shanghai GeneChem Co., Ltd. The wt miR-146a (GV268/miR-146a wt) and miRNA-NC (GV268 empty vector) were constructed, packed and purified by Shanghai GeneChem Co., Ltd. GV268 (CMV-MCS-SV40-Neomycin) plasmid information was presented in Fig. S2. The sequence of hsa-miR-146a was $\left(5^{\prime} \rightarrow 3^{\prime}\right)$ : CCUCUGAAAUUCAGUUCU UCAG.

AD-like model. A total of 12 C57BL/6 male mice were randomly and evenly divided into the control and model groups. AD mice were modelled based on a previously described method (20-22). Mice were shaved with a razor and back hair was removed with depilatory paste $24 \mathrm{~h}$ prior to model establishment. The size of the shaved region was $\sim 2 \times 2 \mathrm{~cm}$. On the first day of experimentation, $0.5 \%$ DNFB solution made with $50 \mu \mathrm{l}$ acetone and olive oil (acetone:olive oil, 4:1) was applied to the back skin of the model group, once, on day 1 . On the 5th day of experimentation, an allergic reaction was induced on the right ear and back using 20 and $50 \mu 10.2 \%$ DNFB solution, respectively. This procedure was repeated every 3 days (on day 8, 11 and 14). The same quantity of acetone-olive-oil solution was administered to the control group without the induction of an allergic reaction (only acetone/olive oil matrix).

Mouse ear thickness measurement. To investigate AD-like the change in ear thickness, ear swelling was observed on the last day of the experiment (day 15). Mouse ear thickness was also measured and recorded using an electronic digital caliper (Guilin Guanglu Measuring Instrument Co., Ltd.).

Histological examination. The dorsal skin samples of mice were harvested and fixed with $10 \%$ neutral-buffered formalin for $48 \mathrm{~h}$ at $4^{\circ} \mathrm{C}$, dehydrated and embedded in paraffin. Then, $4 \mu \mathrm{m}$ sections were stained with hematoxylin for $5 \mathrm{~min}$ and eosin for 1-2 min, then dehydrated in 95\% and absolute alcohols at room temperature. Tissue sections were examined in three different areas (magnification, x200) using an Olympus BX53 light microscope (Olympus Corporation).

Western blotting. The protein samples derived from 293T cells were detected by western blotting. Briefly, the sample was lysed in RIPA buffer (Cell Signaling Technology, Inc.) with protease inhibitor cocktail (Roche Applied Science) and centrifuged at $12,000 \mathrm{x} \mathrm{g}$ for $10 \mathrm{~min}$ at $4^{\circ} \mathrm{C}$. The concentration of protein samples was detected using the BCA protein assay kit (Thermo Fisher Scientific, Inc.). Equal amounts of cellular 
protein $(40 \mu \mathrm{g} /$ lane) were separated by $10 \%$ tris-glycine sodium dodecyl sulfate-polyacrylamide gel electrophoresis, and then transferred onto nitrocellulose filter membranes. Following transfer, the membranes were blocked with Tris-buffered saline (TBS) containing 5\% non-fat milk, and $0.1 \%$ tween (TBST) for $1 \mathrm{~h}$ at room temperature. The membranes were washed three times for $5 \mathrm{~min}$ each with $10 \mathrm{ml}$ of TBST at room temperature, and sequentially incubated with various primary antibodies: GAPDH (diluted in 5\% BSA at 1:1,000, Cell Signaling Technology, Inc., catalog no. 2118), SUMO1 (diluted in 5\% BSA at 1:1,000, Cell Signaling Technology, Inc., catalog no. 4940) overnight at $4^{\circ} \mathrm{C}$. The washing was repeated 3 times, followed by incubation with HRP-conjugated anti-rabbit secondary antibody (diluted in 5\% BSA at 1:5,000, Cell Signaling Technology, Inc., catalog no. 7074) for $1 \mathrm{~h}$ at room temperature. The bands were detected with ECL reagents (Merck KGaA). All data are representatives of three independent experiments. Bands were quantified with densitometry analysis using Gel-Pro Analyzer version 4.0 software (Media Cybernetics, Inc.).

Flow cytometry analysis of Th1 $\left[C D 4^{+}\right.$interferon $\left.(\mathrm{IFN})-\gamma^{+}\right]$ and Th2 $\left[C D 4^{+}\right.$interleukin (IL) $\left.-4^{+}\right]$. For blood sampling, ethylenediamine tetraacetic acid anticoagulant tubes were used to collect left arm venous blood from AD patients. Peripheral blood mononuclear cells (PBMCs) were isolated via discontinuous density gradient centrifugation with Lymphoprep ${ }^{\mathrm{TM}}$ (STEMCELL Technologies) for $20 \mathrm{~min}$ at $800 \mathrm{x} \mathrm{g}$ at room temperature $\left(20-25^{\circ} \mathrm{C}\right)$. PBMCs were placed in a $37^{\circ} \mathrm{C}$ water bath immediately after removal from a liquid nitrogen tank and slowly rotated until samples were fully thawed. According to the manufacturer's protocols of the Th1/Th2 phenotyping kit (BD Biosciences), PBMCs were examined on a cell sorter after cells were resuspended in RPMI1640 medium (Thermo Fisher Scientific, Inc.) supplemented with $10 \%$ fetal bovine serum (Thermo Fisher Scientific, Inc.), then $1 \mathrm{ml}$ of cold fixation buffer (provided in the kit) was added and the cells incubated for $20 \mathrm{~min}$ at room temperature. The cells were permeabilized in 1X Perm/Wash ${ }^{\mathrm{TM}}$ buffer (provided in the kit) and incubated at room temperature for $15 \mathrm{~min}$ then sample antibodies $(20 \mu \mathrm{l})$ added and incubated for $30 \mathrm{~min}$ in the dark at room temperature. The number of $\mathrm{CD}^{+} \mathrm{T}$ cellular gates were measured using a PerCP-Cy5.5-conjugated anti-CD4 antibody (clone SK3, BD Pharmingen; BD Biosciences, catalog no. 560751), with 100,000 CD4 ${ }^{+} \mathrm{T}$ cells for each sample incubated at room temperature for $30 \mathrm{~min}$ in the dark. In addition, samples were stained with fluorescein isothiocyanate (FITC)-labeled anti-IFN $\gamma$ (clone B27, BD Pharmingen; BD Biosciences, catalog no. 560751), allophycocyanin (APC)-anti-IL-4 (clone MP4-25D2, BD Pharmingen; BD Biosciences, catalog no. 560751) and incubated at room temperature for $30 \mathrm{~min}$ in the dark. The percentage of Th1 $\left(\mathrm{CD} 4^{+} \mathrm{IFN}-\gamma^{+}\right)$and $\mathrm{Th} 2\left(\mathrm{CD} 4^{+} \mathrm{IL}-4^{+}\right)$cells was subsequently calculated. Analysis was conducted with a flow cytometer and FACSDiva 7.0 software (BD Biosciences).

Flow cytometry analysis of splenocytes. Murine spleens were acquired under aseptic conditions and ground in a Petri dish. Samples were then centrifuged at 1,500 $\mathrm{x} g$ for $30 \mathrm{~min}$ at room temperature and the supernatant discarded. Red blood cell lysis buffer (5 ml, BD Biosciences, catalog no. 555899) was added to samples and the mixture was incubated in the dark for $15 \mathrm{~min}$ at $37^{\circ} \mathrm{C}$. The mixture was subsequently centrifuged at $200 \mathrm{x} \mathrm{g}$ for $5 \mathrm{~min}$ at room temperature a second time and the supernatant discarded. Similar to flow cytometry analysis of PBMCs, spleen cells were examined for expression of Th1 and Th2 according to the manufacturer's instructions (BD Biosciences, catalog no. 560758). The number of $\mathrm{CD}^{+}{ }^{+} \mathrm{T}$ cellular gates was measured using FITC-conjugated anti-CD4 antibodies (clone RM4-5, provided in the kit), with 100,000 CD4+T cells for each sample. They were stained with PerCP-Cy5.5-labeled anti-IFN $\gamma$ (clone XMG1.2, provided in the kit) and APC-anti-IL-4 (clone 11B11, provided in the kit) incubated at room temperature for $30 \mathrm{~min}$ in the dark. The percentage of Th1 $\left(\mathrm{CD} 4^{+} \mathrm{IFN}-\gamma^{+}\right)$and Th2 $\left(\mathrm{CD} 4^{+} \mathrm{IL}-4^{+}\right)$cells was then calculated. Flow cytometry data was analyzed with FACSDiva 7.0 software.

Reverse transcription quantitative $(R T-q) P C R$ analysis of miR-146a human serum expression. miR-146a extracted from serum of patients with AD and healthy controls and total RNA was extracted using the QIAGEN miRNeasy serum/plasma kit (Qiagen GmbH, catalog no. 217184) and the First strand cDNA synthesis kit (Thermo Fisher Scientific, Inc.), respectively. Serum $(220 \mu \mathrm{l})$ was obtained after transferring the sample from $-80^{\circ} \mathrm{C}$ storage and thawing on ice. miRNA was then extracted from the serum using aforementioned methods. Reagents were added during the RT process according to the manufacturer's protocols (Qiagen $\mathrm{GmbH}$, catalog no. 217184); qPCR was performed with an ABI Prism 7500 PCR system (Applied Biosystems; Thermo Fisher Scientific, Inc.). The miR-146a and miR-39 primers were synthesized by Guangzhou RiboBio Co., Ltd. and due to the company's patents, specific primer sequences cannot be given. Samples were amplified under the following conditions: $95^{\circ} \mathrm{C}$ for $10 \mathrm{~min}$, followed by 45 cycles of $95^{\circ} \mathrm{C}$ for $15 \mathrm{sec}$ and $60^{\circ} \mathrm{C}$ for $1 \mathrm{~min}$. To calculate the expression of the target miR-146a (Bulge-Loop miRNA-146a reverse transcription-primers, Guangzhou RiboBio Co., Ltd.) relative to suitable reference gene (Bulge-Loop miRNA-39 reverse transcription-primers, Guangzhou RiboBio Co., Ltd.). The expression of miR-146a was calculated using the $2^{-\Delta \Delta C q}$ method (23).

$R T-q P C R$ analysis of miR-146a expression in mouse skin. Back skin samples from mice were collected and transferred to a refining tube. TRIzol $(1 \mathrm{ml})$ was added and total RNA was extracted. RNA concentrations were quantified using a UV spectrophotometer and RT was performed with 1,000 ng RNA in accordance with the manufacturer's protocols. qPCR was performed based on the corresponding kit procedures (Guangzhou RiboBio Co., Ltd.) and the expression of miR-146a was calculated using the $2^{-\Delta \Delta \mathrm{Cq}}$ method (23). The amplification program consisted of 1 cycle of $95^{\circ} \mathrm{C}$ for $10 \mathrm{~min}$, followed by 45 cycles of $95^{\circ} \mathrm{C}$ for $15 \mathrm{sec}$ and $60^{\circ} \mathrm{C}$ for $1 \mathrm{~min}$. The expression of miR-146a (Bulge-Loop miRNA-146a reverse transcription-primers, Guangzhou RiboBio Co., Ltd.) relative to the expression of the miR-39 (Bulge-Loop miRNA-39 reverse transcription-primers, Guangzhou RiboBio Co., Ltd.) used as a reference gene.

miRNA sequencing and target prediction. miRNA analysis was performed using the Illumina HiSeq 4000 sequencing platform (Illumina, Inc.). TargetScan (version 7.2, 

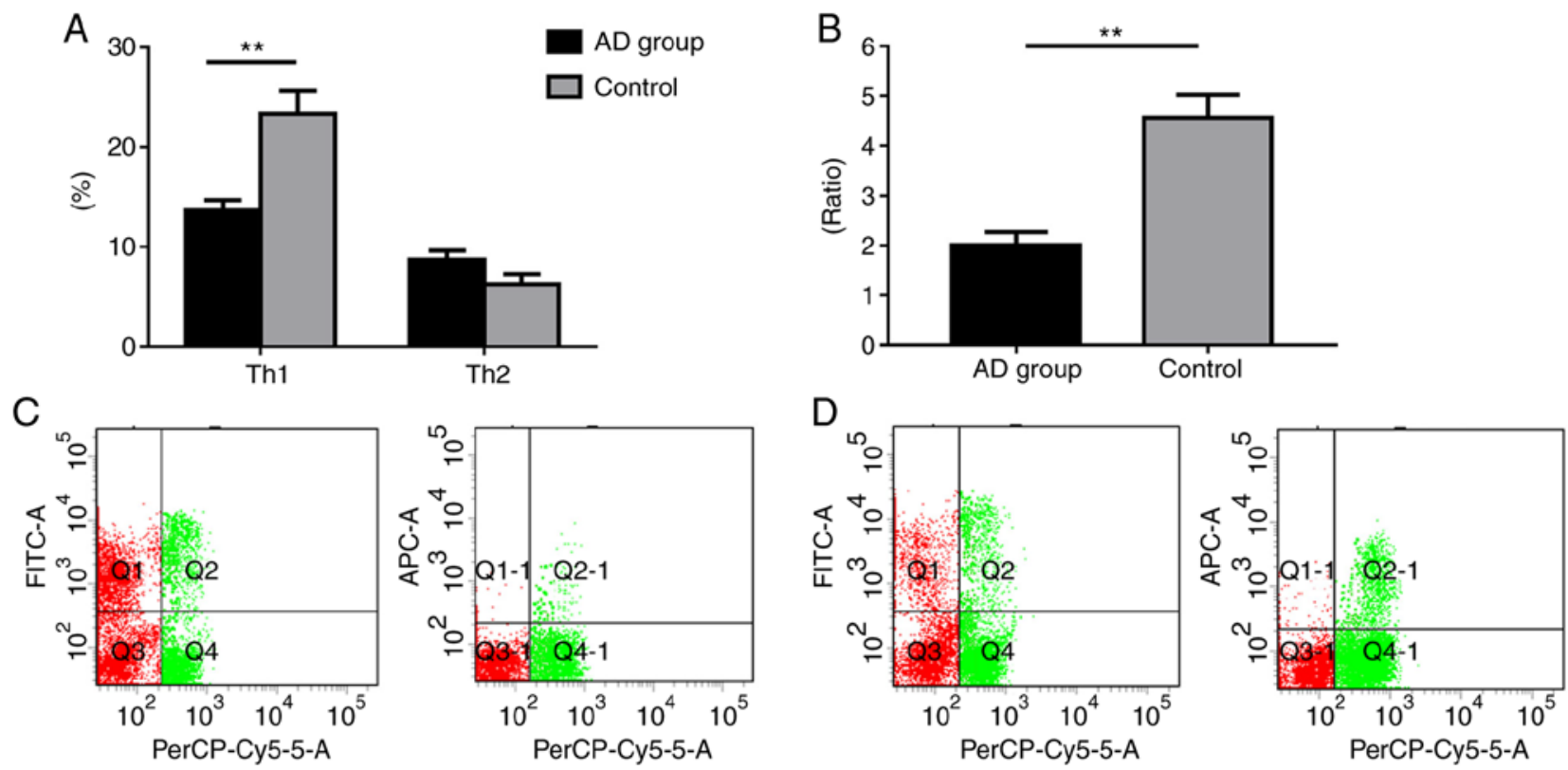

Figure 1. Th1/Th2 ratio expression profiling of patients with AD and healthy controls. (A) Percentage of Th1 and Th2 cells. (B) Th1/Th2 ratio. (C) Control group flow cytometry analysis. (D) Flow cytometry analysis of patients with AD. PerCP-Cy5.5, FITC, and APC denote CD4 $4^{+}$, Th1 (CD4 IFN- $\left.\gamma^{+}\right)$, and Th2 $\left(\mathrm{CD} 4^{+} \mathrm{IL}-4^{+}\right)$, respectively. Data are presented as the mean \pm SEM (AD group, $\mathrm{n}=25$; Control group, $\mathrm{n}=16$ ). ${ }^{* *} \mathrm{P}<0.01$. Th, T-helper; IFN, interferon; IL, interleukin; FITC, fluorescein isothiocyanate; AD, atopic dermatitis.

A

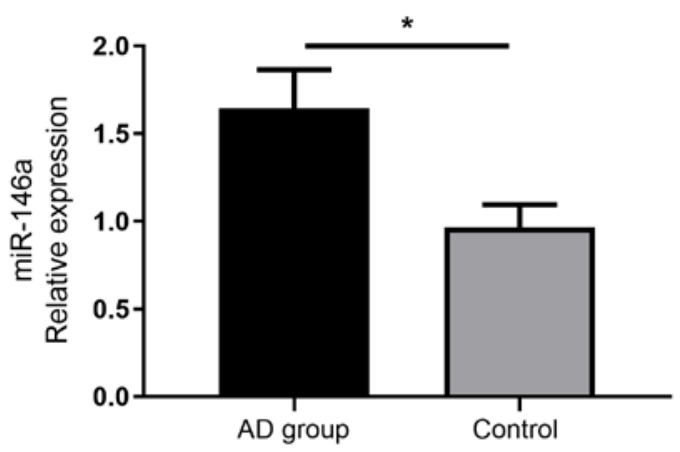

B

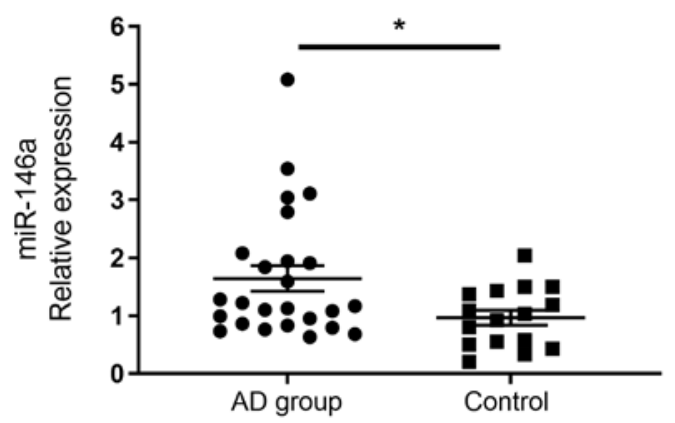

Figure 2. miR-146a expression in the serum of patients with AD compared with the control group. Reverse transcription-quantitative PCR analysis of miR-146 in the serum of healthy controls and AD patients. (A) Histogram. (B) Scatter plot. Data are presented as the mean \pm standard error of the mean (AD group, $\mathrm{n}=25$; Control group, $\mathrm{n}=16$ ). ${ }^{*} \mathrm{P}<0.05$. miR, microRNA; AD, atopic dermatitis.

http://www.targetscan.org/vert_72/) (24) were utilized to predict the target gene of miR-146a. In accordance with a recent study (25), it was predicted that SUMO1 was a target gene of miR-146a (25).

Transfection and luciferase assays. 293T cells (Cell Bank of Type Culture Collection of Chinese Academy of Sciences) were transfected using the X-tremeGENE ${ }^{\mathrm{TM}}$ HP DNA transfection reagent (Roche Diagnostics) in accordance with the manufacturer's protocols. Dual-luciferase assays were performed with $0.1 \mu \mathrm{g}$ 3'UTR luciferase plasmids, $0.4 \mu \mathrm{g}$ miRNA expression plasmids and $0.02 \mu \mathrm{g}$ Renilla plasmids in a 24 -well plate at a density of $1 \times 10^{5}$ cells/well. Dual-luciferase assays were conducted at $48 \mathrm{~h}$ post-transfection using the Dual-Glo Luciferase assay system according to the manufacturer's protocols (Promega Corporation). Luciferase readings were corrected for background, and Firefly luciferase values were used to normalized Renilla luciferase activity. We constructed 3'UTR-NC (3'-UTR GV272 empty vector), 3'UTR-MU (3'-UTR GV272/SUMO1 mu), 3'UTR (3'-UTR GV272/SUMO1 wt), as well as miRNA-NC (GV268 empty vector) and miRNA (GV268/miR-146a wt) plasmids. RT-qPCR analysis (as aforementioned) was also performed on $293 \mathrm{~T}$ cells transfected with GV268/miR-146a and GV268 empty vector (negative control), which demonstrated significant upregulation of miR-146a in the cells transfected with GV268/miR-146a compared with the non-transfected control cells as a negative control group (Fig. S3).

Statistical analysis. Statistical analyses were performed using SPSS 17.0 software (SPSS, Inc.) and data are presented as the mean + standard error of the mean. If group data were normally distributed, an independent t-test was performed. Otherwise data were analyzed using a Mann-Whitney U test. One-way analysis of variance was used for comparing the data of three or more groups; a Dunnett's post-hoc test was 

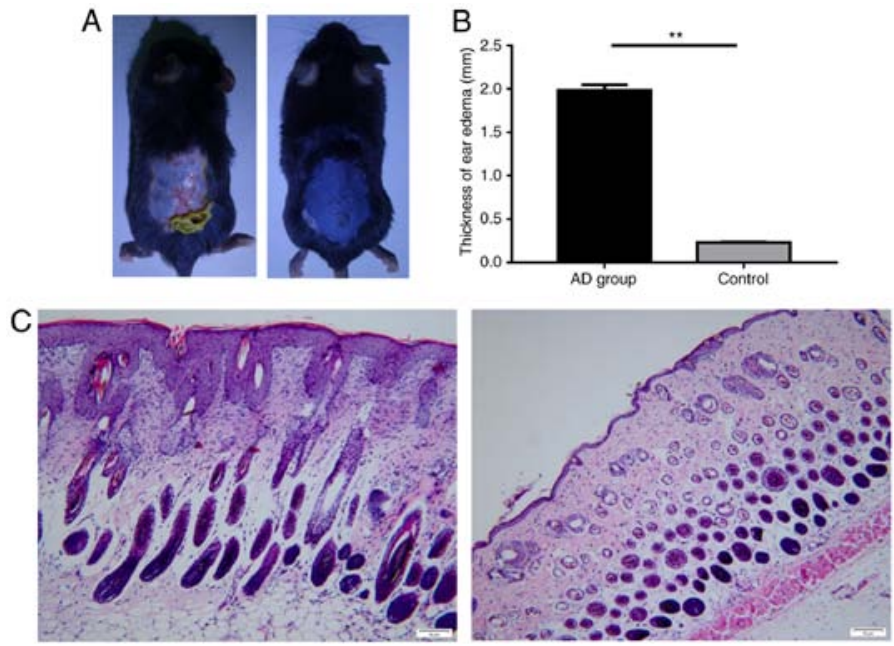

Figure 3. Histological features of AD-like skin in C57BL/6 mice. (A) Images were taken on day 14. Mouse back skin of the control group (left) vs. the model group. (B) The degree of ear swelling in the model group revealed a statistically significant increase in AD mice compared with the control group. (C) Histology of back skin. Mouse skin tissue pathology in the control group (left) and model group (right). Magnification, x200 (n=6 per group). Scale bar=50 $\mu \mathrm{m}$. Data are presented as the mean \pm standard error of the mean. ${ }^{* *} \mathrm{P}<0.01$. AD, atopic dermatitis.
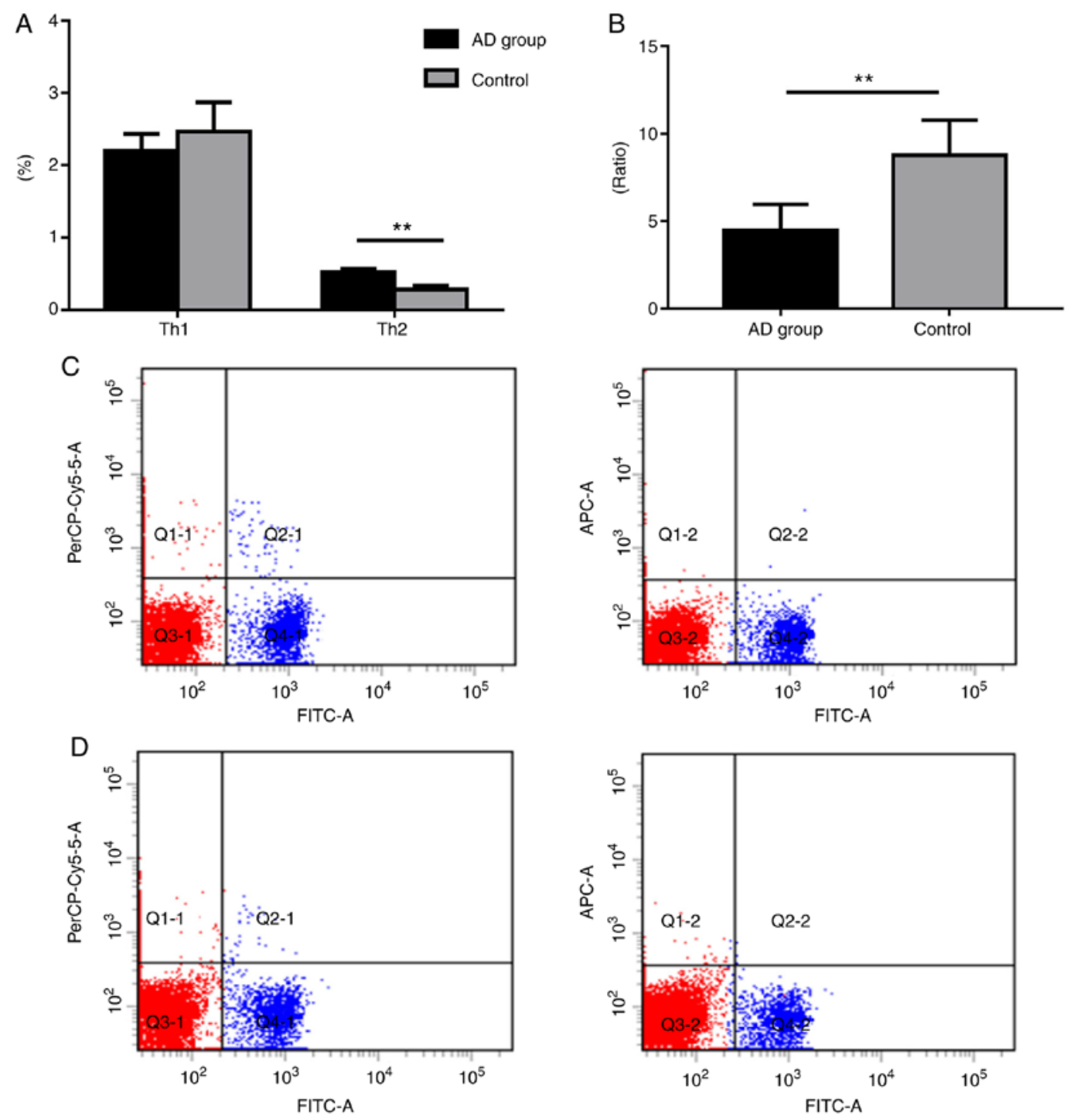

Figure 4. Expression profiles of Th1 and Th2 cells in murine spleens. (A) Th1 (CD4 $\left.4^{+} \mathrm{IFN}-\gamma^{+}\right)$and Th2 $\left(\mathrm{CD} 4^{+} \mathrm{IL}-4^{+}\right)$cell percentage analysis. (B) Th1/Th2 ratio Th1 and Th2 flow cytometry for the (C) control and (D) model groups. FITC, PerCP-Cy5.5, and APC denote CD4 $4^{+}$, Th1 (CD4+IFN- $\left.\gamma^{+}\right)$and Th2 (CD4+IL- $\left.4^{+}\right)$, respectively. Data are presented as the mean \pm SEM $(n=6)$. ${ }^{* *} \mathrm{P}<0.01$. Th, T-helper; IFN, interferon; IL, interleukin; FITC, fluorescein isothiocyanate; AD, atopic dermatitis. 
A

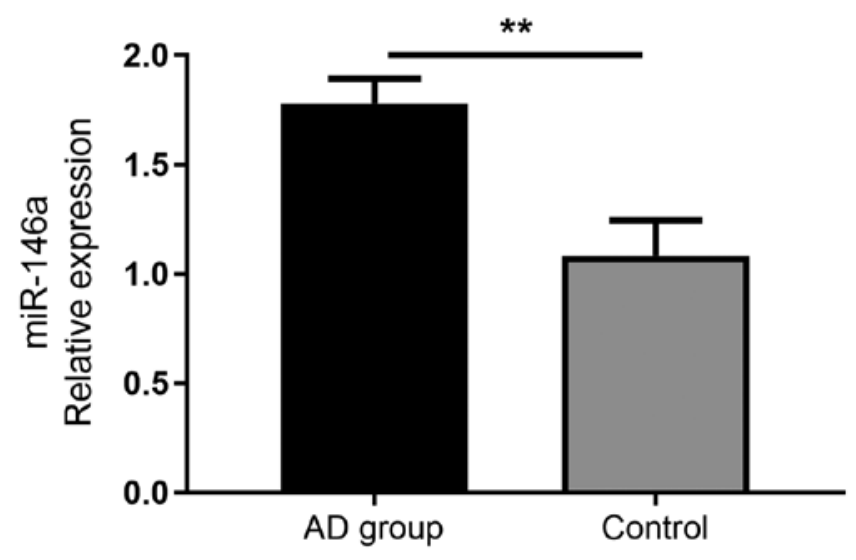

B

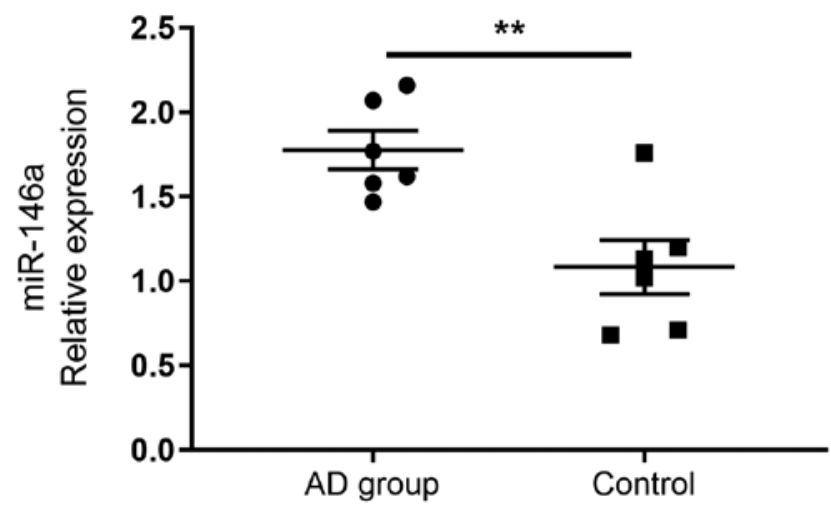

Figure 5. Reverse transcription-quantitative PCR analysis of miR-146 expression in mouse skin. The experimental results revealed that the levels of miR-146a expression in the model group were significantly increased compared with the control group. (A) Histogram. (B) Scatter plot. Data are presented as the mean \pm standard error of the mean $(n=6) .{ }^{* *} \mathrm{P}<0.01$. miR microRNA; AD, atopic dermatitis.

also conducted. $\mathrm{P}<0.05$ was considered to indicate a statistically significant difference.

\section{Results}

Clinical characteristics of healthy controls and patients with $A D$. To assess the role of miR-146a in the serum of patients with $\mathrm{AD}$, Th1/Th2 ratio expression profiling was performed in healthy individuals and AD patients. The results revealed that the AD group exhibited significantly decreased Th1 levels compared with the control group $(\mathrm{P}<0.01)$. Patients with AD also exhibited increased Th2 levels, although the difference was not statistically significant $(\mathrm{P}>0.05)$. Additionally, our results demonstrated that the AD group exhibited a significantly lower Th1/Th2 ratio compared with the control $(\mathrm{P}<0.05$; Fig. 1).

miR-146a is upregulated in the serum of patients with AD. As miR-146a has been demonstrated to serve immune regulatory functions, previous reports have confirmed its increased expression in the non-lesional and chronic lesional skin of patients with AD (14). However, to the best of our knowledge, no studies have assessed the expression of miR-146a in the serum of patients with AD. The current study demonstrated that miR-146a expression was significantly increased in the serum of patients with AD than in healthy individuals (Fig. 2).

DNFB-induces AD-like skin inflammation in C57BL/6 mice. To determine whether miR-146a expression serves a role in AD animal models, AD-like skin inflammation was induced via DNFB in a mouse model. The results revealed that the skin of the control mice appeared to be normal, while the skin of the model group was thicker, roughened and scarred. Cutaneous ulcers, erythema and desquamation also developed (Fig. 3A). Furthermore, the degree of ear swelling induced by DNBF in C57BL/6 mice was significantly higher in the AD group than in the control (Fig. 3B). In addition, the skin tissue pathology of the control group indicated no apparent abnormality, yet the model group exhibited notable pathological changes, including epidermal parakeratosis, hyperkeratosis, spongiosis, acanthosis and excessive lymphocyte infiltration in the dermis (Fig. 3C).

Shift of the Th1/Th2 ratio in murine spleens. The Th1 $\left(\mathrm{CD} 4^{+} \mathrm{IFN}-\gamma^{+}\right)$levels of the model group were markedly decreased compared with the control group; however, the count of Th2 (CD4 $\left.{ }^{+} \mathrm{IL}-4^{+}\right)$cells in the model group was significantly higher when compared with the control group (Fig. 4). Furthermore, the ratio of Th1/Th2 was significantly lower in the model group compared with control group $(\mathrm{P}<0.01$; Fig. 4).

miR-146a expression in mouse skin. To investigate the expression of miR-146a during skin inflammation, the expression of miR-146a was determined in mouse skin using a DNFB-induced AD-like model. The results revealed significantly upregulated miR-146a expression in the model group compared with the control group (Fig. 5). This result was consistent with the expression of miR-146a in the serum of the patients with AD.

miR-146a affects SUMO1 expression. Second-generation sequencing and a combined literature analysis was performed in the current study. The results revealed that the potential molecular target of miR-146a was SUMO1 (21). To functionally determine whether miR-146a has a direct effect on SUMO1 activation, vectors encoding a partial sequence of the 3'UTR of SUMO1 mRNA were utilized, where the predicted miR-146a target sites were located (Fig. 6A). The results revealed that luciferase activity significantly decreased following co-transfection with miR-146a and the vector carrying the 3'UTR of SUMO1 compared with the negative control (Fig. 6B). Then, we evaluated the protein expression levels of SUMO1 in 293T cells transfected with miR-146a and negative control. As presented in Fig. 6C, the quantification of band density of SUMO1 expression was not statistically different in each group.

\section{Discussion}

$\mathrm{AD}$ is a common skin disease that causes pruritic and chronically relapsing inflammation in children and adults $(26,27)$. miRNAs are relatively novel molecules that have been widely 
A

\begin{tabular}{|c|c|c|c|c|c|c|c|}
\hline & $\begin{array}{c}\text { Predicted consequential pairing } \\
\text { of target region (top) and miRNA (bottom) }\end{array}$ & $\begin{array}{l}\text { Site } \\
\text { type }\end{array}$ & $\begin{array}{c}\text { Context++ } \\
\text { score }\end{array}$ & $\begin{array}{l}\text { Context++ } \\
\text { score percentile }\end{array}$ & $\begin{array}{c}\text { Weighted } \\
\text { context++score }\end{array}$ & $\begin{array}{l}\text { Conserved } \\
\text { branch length }\end{array}$ & $\mathrm{P}_{\mathrm{CT}}$ \\
\hline $\begin{array}{l}\text { Position } 400-407 \text { of } \\
\text { SUMO1 3' UTR } \\
\text { hsa-miR-146a-3p }\end{array}$ & $\begin{array}{l}5^{\prime} \text {...GGGAGAUGGAAGUUUUUCAGAGA... } \\
\text { | }|||||| \mid \\
3^{\prime} \quad \text { GACUUCUUGACUUAAAGUCUCC }\end{array}$ & 8 mer & -0.35 & 98 & -0.35 & 0 & N/A \\
\hline
\end{tabular}
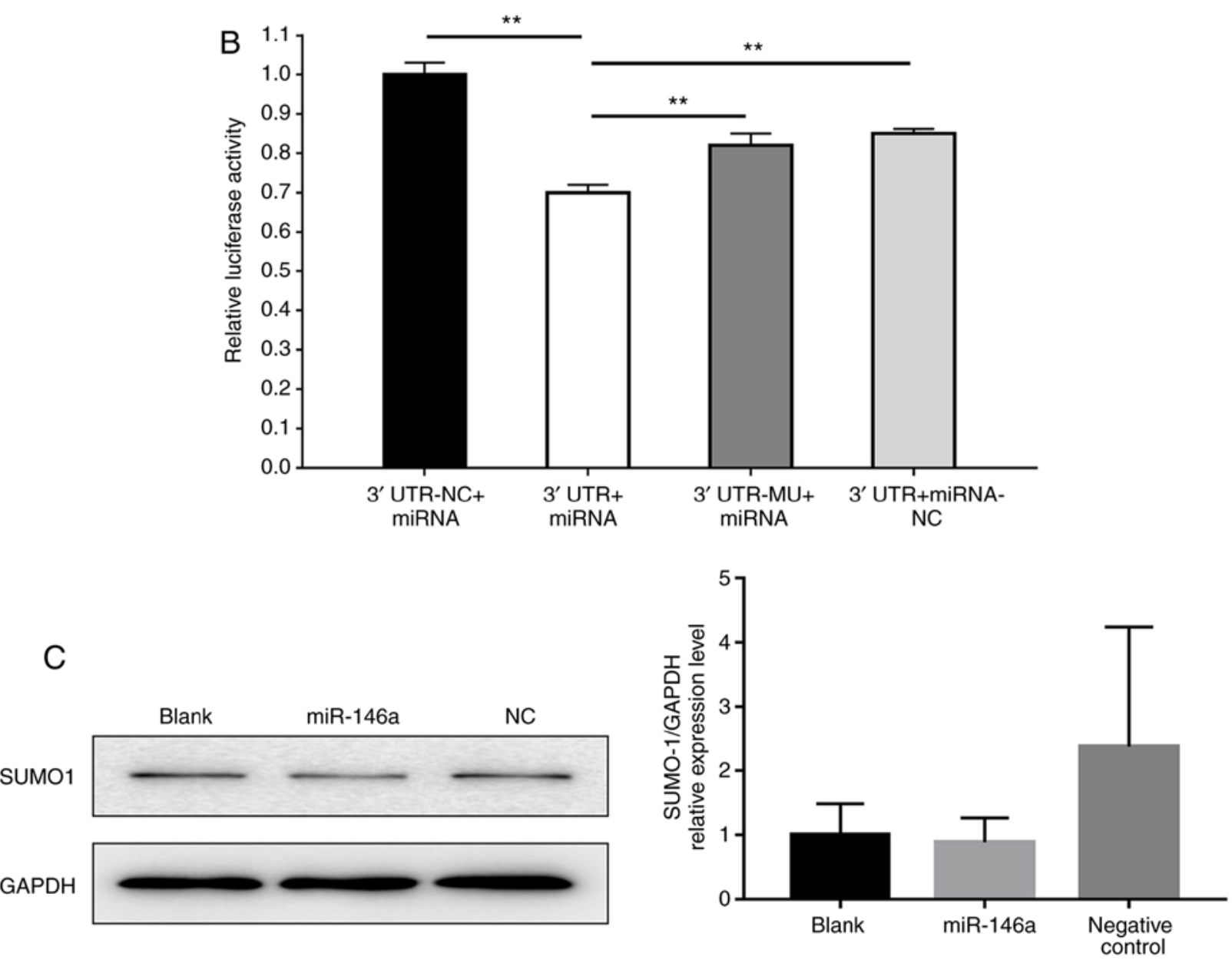

Figure 6. miR-146a affects SUMO1 expression. (A) Bioinformatic analysis indicated the prediction of the complementary site of miR-146a in the SUMO1 3'-UTR (TargetScan). (B) Firefly luciferase activity was used to normalize the luciferase activity of Renilla following co-transfection, and subsequently normalized for SUMO1 expression in the presence of miR-146a. Values are presented as the relative luciferase activity \pm the standard deviation of three independent experiments. For each 3'UTR construct, normalized luciferase activity in the absence of miR-146a was set to $1{ }^{* * *} \mathrm{P}<0.01$. (C) Western blot analysis the protein expression of SUMO1 in 293T cells transfected with miR-146a and negative control. Data are representative of three independent experiments. 3'UTR-NC + miRNA, 3'-UTR GV272 empty vector + GV268/miR-146a wt group; 3'UTR + miRNA, 3'-UTR GV272/SUMO1 wt + GV268/miR-146a wt group; 3'UTR-MU + miRNA, 3'-UTR GV272/SUMO1 mu + GV268/miR-146a wt group; 3'UTR + miRNA-NC, 3'-UTR GV272/SUMO1 wt + GV268 empty vector group; miRNA/miR, microRNA; SUMO1, small ubiquitin-related modifier 1; 3'-UTR, 3'-untranslated region; NC, negative control; mu, mutant; wt, wild type.

studied in recent years to determine their exact function in the human body (28). However, information regarding the role of miRNAs in AD is limited. Recent studies have described an altered expression of miRNAs in the skin or serum of patients with AD (29,30). Psoriatic AD patients have been characterized by the reduced expression of miR-122a, miR-133a-133b, miR-133b, miR-215 and miR-326, whereas the downregulated expression of miR-33, miR-483, miR-515-5p and miR-519d has occurred only in patients with AD (31). In addition, 10 upregulated and 34 downregulated miRNAs have been identified in the affected skin of patients with AD (32). It has been indicated that miRNAs control an early phase of inflammation by delaying the expression of cytotoxic T-lymphocyte-associated protein 4 (33). Specifically, multiple CD $4^{+}$helper cells, including Th1, Th2, Th9, Th17, Th22 and T regulatory (Treg) cells, as well as their associated cytokines, participate in the onset of disease. These cells interact with, control and affect each other, forming a complex cellular and cytokine network that alters immunoregulation (34). The classic pathway of AD development states that the imbalance of the two major types of $\mathrm{CD} 4^{+}$helper T cells, Th1 and Th2, causes a dominant Th2-type immune response. The key feature is the bidirectional polarization of $\mathrm{T}$ cells $(35,36)$. In the acute phase, IL-4-expressing Th 2 cells dominate and in the chronic phase, IFN- $\gamma$-expressing Th1 cells serve a major role $(37,38)$. The current study revealed a decrease in Th1, an increase in Th2 and an imbalance in the Th1/Th2 ratio in the PBMCs of patients with AD, which is in agreement with the classic pathway of $\mathrm{AD}$ development. 
In addition, flow cytometry analysis of spleen cells in a mouse AD model demonstrated a statistically significant decrease in Th1 cells, an increase in Th2 cells and a decrease in the Th1/Th2 ratio of mice in the model group compared with the control group. This model is in accordance with the classic pathway in human AD, in which an imbalance in Th1/Th2 was reported to cause a dominant Th2-type immune response (36). The pathological characteristics and skin lesions of mice may therefore similar to that of AD in humans. Hence, effective animal models could be used for studying the mechanisms of $\mathrm{AD}$ and developing novel therapeutic strategies.

A previous study demonstrated that miR-146a serves a major role in certain physiological and pathological processes, including the onset and development of immunity, tumors and inflammation (39). The abnormal expression of miR-146a is closely associated with systemic lupus erythematosus, psoriasis, rheumatoid arthritis, tumors and asthma (40-43). High expression of miR-146a in Treg cells greatly contributes to the maintenance of normal cell immunoregulatory functions (44). Through regulating its target genes, including tumor necrosis factor receptor associated 6, IL1 receptor associated kinase 1 , IFN regulatory factor 5, and signal transducer and activator of transcription 1, miR-146a participates in the development of many diseases $(45,46)$. A recent study indicated that miR-146a negatively regulates osteogenesis and bone regeneration from adipose-derived mesenchymal stem cells (47). Furthermore, it has been suggested that miR-146a alleviates chronic skin inflammation in AD by controlling nuclear factor- $\kappa \mathrm{B}$-dependent signaling inflammatory responses in keratinocytes (17). Rebane et al (17) revealed that certain molecules and cytokines are regulated by miR-146a, in which miR-146a-deficient mice were used. It was demonstrated that stronger inflammatory features were exhibited in these miR-146a-deficient mice (17). Compared with the aforementioned study, we reported a potential target gene of miR-146a as SUMO1. Furthermore, miR-146a may be considered as a potential regulator involved in the pathogenesis of AD. It has also been revealed that miR-146a induced cardiac dysfunction in maladaptive hypertrophy and suppressed SUMO1 expression in heart failure (25). In the present study, increased expression of miR-146a was observed in the serum of patients with AD and in the skin of model group mice, indicating that miR-146a may participate in the development of $\mathrm{AD}$; however, further studies are required to reveal its underlying mechanisms.

The current study analyzed the expression of miR-146a in patients with AD and in an animal model of AD. The limitations of the study include the few patients with AD that were enrolled. A larger sample size may better reflect whether miR-146a is a novel target for drug intervention. Our results demonstrated that the C57BL/6 mouse AD model may reflect the classic process of human AD (48), in which an imbalance in Th1/Th2 causes a dominant Th2-type immune response. The results of luciferase assay in the present study indicated that the potential direct target of miR-146a may be SUMO1. Therefore, it could be considered to be an appropriate model for AD studies. Increased expression of miR-146a in the serum of patients with AD and the skin of model group mice indicates that miR-146a may be involved in the development of $\mathrm{AD}$ and that SUMO1 could be a potential direct mRNA target of miR-146a. Further investigation into its regulatory mechanisms is required, particularly in the immune regulation of AD, such as T cell regulation and Th1/Th2 balance. In conclusion, miR-146a may be a novel target for drug intervention in the treatment of patients with AD.

\section{Acknowledgements}

Not applicable.

\section{Funding}

The current study was supported by the National Natural Science Foundation of China (grant no. 81774307), the Specific Research Fund for TCM Science and Technology of Guangdong Provincial Hospital of Chinese Medicine (grant nos. YN2015MS06, YN2015QN09 and YN2016QJ11), the Traditional Chinese Medicine Bureau of Guangdong Province (grant no. 20183004) and the Department of Science and Technology of Guangdong Province [grant nos. (2017)105 and 2017A030310122)].

\section{Availability of data and materials}

The datasets used and/or analyzed during the current study are available from the corresponding author on reasonable request.

\section{Authors' contributions}

DC and YL designed the experiments, supervised all research and revised the manuscript. FY and WM conducted the experiments and wrote the manuscript. SY, XZ, XM and JL conducted the experiments and contributed to the interpretation of data. All authors read and approved the final version.

\section{Ethics approval and consent to participate}

The use of human samples was approved by the Ethics Committee of Guangdong Provincial Hospital of Chinese Medicine (approval no. B2015-017-01). Written informed consent was obtained from each participant prior to enrollment. The care and use of laboratory animals was performed in accordance to the Laboratory Animal Research Ethical Committee Guidelines of Guangdong Provincial Hospital of Chinese Medicine. The present study was approved by the Laboratory Animal Ethics Committee of Guangdong Provincial Hospital of Chinese Medicine, approval number: 2015016).

\section{Patient consent for publication}

Not applicable.

\section{Competing interests}

The authors declare that they have no competing interests.

\section{References}

1. Weidinger S, Beck LA, Bieber T, Kabashima K and Irvine AD: Atopic dermatitis. Nat Rev Dis Primers 4: 1, 2018.

2. Bieber T: Atopic dermatitis. N Engl J Med 358: 1483-1494, 2008. 
3. Xu F, Yan S, Li F, Cai M, Chai W, Wu M, Fu C, Zhao Z, Kan H, Kang K and Xu J: Prevalence of childhood atopic dermatitis: An urban and rural community-based study in shanghai, China. PLoS One 7: e36174, 2012.

4. Guo Y, Li P, Tang J, Han X, Zou X, Xu G, Xu Z, Wei F, Liu Q, Wang M, et al: Prevalence of atopic dermatitis in Chinese children aged 1-7ys. Sci Rep 6: 29751, 2016.

5. Wang $\mathrm{AX}$ and $\mathrm{Xu}$ Landén $\mathrm{N}$ : New insights into $\mathrm{T}$ cells and their signature cytokines in atopic dermatitis. IUBMB Life 67 601-610, 2015

6. Li S, Liu L, Zhuang X, Yu Y, Liu X, Cui X, Ji L, Pan Z, Cao X, Mo B, et al: MicroRNAs inhibit the translation of target mRNAs on the endoplasmic reticulum in arabidopsis. Cell 153: 562-574, 2013.

7. Wahid F, Shehzad A, Khan T and Kim YY: MicroRNAs: Synthesis, mechanism, function, and recent clinical trials. Biochim Biophys Acta 1803: 1231-1243, 2010.

8. Lau PW and MacRae IJ: The molecular machines that mediate microRNA maturation. J Cell Mol Med 13: 54-60, 2009.

9. Sonkoly E and Pivarcsi A: Advances in microRNAs: Implications for immunity and inflammatory diseases. J Cell Mol Med 13: 24-38, 2009.

10. Yoshizawa JM and Wong DT: Salivary microRNAs and oral cancer detection. Methods Mol Biol 936: 313-324, 2013.

11. Tili E, Michaille JJ, Wernicke D, Alder H, Costinean S, Volinia S and Croce CM: Mutator activity induced by microRNA-155 (miR-155) links inflammation and cancer. Proc Natl Acad Sci USA 108: 4908-4913, 2011.

12. West C and McDermott MF: Effects of microRNA-146a on the proliferation and apoptosis of human osteochondrocytes by targeting TRAF6 through the NF- $\kappa \mathrm{B}$ signalling pathway. Biosci Rep 37: pii: BSR20170180, 2017

13. Park H, Huang X, Lu C, Cairo MS and Zhou X: MicroRNA-146a and microRNA-146b regulate human dendritic cell apoptosis and cytokine production by targeting TRAF6 and IRAK1 proteins. J Biol Chem 290: 2831-2841, 2015.

14. Lindner JM, Kayo H, Hedlund S, Fukuda Y, Fukao T and Nielsen PJ: Cutting Edge: The transcription factor Bob1 counteracts $\mathrm{B}$ cell activation and regulates miR-146a in B cells. J Immunol 192: 4483-4486, 2014.

15. Wang S, Zhang X, Ju Y, Zhao B, Yan X, Hu J, Shi L, Yang L, Ma Z, Chen L, et al: MicroRNA-146a feedback suppresses T cell immune function by targeting Statl in patients with chronic Hepatitis B. J Immunol 191: 293-301, 2013.

16. Williams AE, Perry MM, Moschos SA, Larner-Svensson HM and Lindsay MA: Role of miRNA-146a in the regulation of the innate immune response and cancer. Biochem Soc Trans 36 $1211-1215,2008$

17. Rebane A, Runnel T, Aab A, Maslovskaja J, Rückert B, Zimmermann M, Plaas M, Kärner J, Treis A, Pihlap M, et al: MicroRNA-146a alleviates chronic skin inflammation in atopic dermatitis through suppression of innate immune responses in keratinocytes. J Allergy Clin Immunol 134: 836-847.el1, 2014.

18. M HJ and Georg R: Diagnostic features of atopic dermatitis. Acta Dermatovener 60: 44-47, 1980

19. Eichenfield LF, Lucky AW, Boguniewicz M, Langley RG Cherill R, Marshall K, Bush C and Graeber M: Safety and efficacy of pimecrolimus (ASM 981) cream 1\% in the treatment of mild and moderate atopic dermatitis in children and adolescents. J Am Acad Dermatol 46: 495-504, 2002.

20. Heo JC, Nam DY, Seo MS and Lee SH: Alleviation of atopic dermatitis-related symptoms by Perilla frutescens Britton. Int J Mol Med 28: 733-737, 2011.

21. Heo JC, Son HU, Kim SL and Lee SH: A derivative of L-allo threonine alleviates 2,4-dinitrofluorobenzene-induced atopic dermatitis indications. Biosci Biotechnol Biochem 76: 2021-2025, 2012.

22. Nam DY, Lee JM, Heo JC and Lee SH: Mitigation of 2,4-dinitrofluorobenzene-induced atopic dermatitis-related symptoms by Terminalia chebula Retzius. Int J Mol Med 28: 1013-1018, 2011.

23. Livak KJ and Schmittgen TD: Analysis of relative gene expression data using real-time quantitative PCR and the 2(-Delta Delta $\mathrm{C}(\mathrm{T})$ ) method. Methods 25: 402-408, 2001

24. Agarwal V, Bell GW, Nam JW and Bartel DP: Predicting effective microRNA target sites in mammalian mRNAs. Elife: 4, 2015 doi: $10.7554 /$ eLife.05005.

25. Oh JG, Watanabe S, Lee A, Gorski PA, Lee P, Jeong D, Liang L, Liang Y, Baccarini A, Sahoo S, et al: miR-146a suppresses SUMO1 expression and induces cardiac dysfunction in maladaptive hypertrophy. Circ Res 123: 673-685, 2018.
26. Deleanu D and Nedelea I: Biological therapies for atopic dermatitis: An update. Exp Ther Med 17: 1061-1067, 2019.

27. Thomsen SF: Atopic dermatitis: Natural history, diagnosis, and treatment. ISRN Allergy 2014: 354250, 2014.

28. Rożalski M, Rudnicka L and Samochocki Z: MiRNA in atopic dermatitis. Postepy Dermatol Alergol 33: 157-162, 2016.

29. Specjalski K and Jassem E: MicroRNAs: Potential biomarkers and targets of therapy in allergic diseases? Arch Immunol Ther Exp (Warsz) 67: 213-223, 2019.

30. Bin L and Leung DY: Genetic and epigenetic studies of atopic dermatitis. Allergy Asthma Clin Immunol 12: 52, 2016

31. Sonkoly E, Wei T, Janson PC, Sääf A, Lundeberg L, Tengvall-Linder $M$, Norstedt $G$, Alenius H, Homey B, Scheynius A, et al: MicroRNAs: Novel regulators involved in the pathogenesis of psoriasis? PLoS One 2: e610, 2007.

32. Bhardwaj N: MicroRNAs in atopic dermatitis: A review. J Transl Genet Genom 1: 15-22, 2017.

33. Sonkoly E, Janson P, Majuri ML, Savinko T, Fyhrquist N, Eidsmo L, Xu N, Meisgen F, Wei T, Bradley M, et al: MiR-155 is overexpressed in patients with atopic dermatitis and modulates T-cell proliferative responses by targeting cytotoxic $\mathrm{T}$ lymphocyte-associated antigen 4. J Allergy Clin Immunol 126: 581-589. e1-e20, 2010

34. Auriemma M, Vianale G, Amerio P and Reale M: Cytokines and T cells in atopic dermatitis. Eur Cytokine Netw 24: 37-44, 2013.

35. Lipozencić J, Pastar Z, Kulisić SM and Pavić I: Immunologic aspects of atopic dermatitis. Acta Dermatovenerol Croat 17: 226-234, 2009.

36. Agrawal R, Wisniewski JA and Woodfolk JA: The role of regulatory T cells in atopic dermatitis. Curr Probl Dermatol 41: $112-124,2011$

37. Miraglia del Giudice M, Decimo F, Leonardi S, Maioello N, Amelio R, Capasso A, Capristo C and Capristo AF: Immune dysregulation in atopic dermatitis. Allergy Asthma Proc 27: 451-455, 2006

38. Grewe M, Bruijnzeel-Koomen CA, Schöpf E, Maioello N, Amelio R, Capasso A, Capristo C and Capristo AF: A role for Th1 and Th2 cells in the immunopathogenesis of atopic dermatitis. Immunol Today 19: 359-361, 1998

39. Li L, Chen XP and Li YJ: MicroRNA-146a and human disease. Scand J Immunol 71: 227-231, 2010.

40. Ammari M, Jorgensen C and Apparailly F: Impact of microRNAs on the understanding and treatment of rheumatoid arthritis. Curr Opin Rheumatol 25: 225-233, 2013.

41. Dai Y, Huang YS, Tang M, Lv TY, Hu CX, Tan YH, Xu ZM and Yin YB: Microarray analysis of microRNA expression in peripheral blood cells of systemic lupus erythematosus patients. Lupus 16: 939-946, 2007.

42. Bartel DP: MicroRNAs: Genomics, biogenesis, mechanism, and function. Cell 116: 281-297, 2004.

43. Jiménez-Morales S, Gamboa-Becerra R, Baca V, Del Río-Navarro BE, López-Ley DY, Velázquez-Cruz R, Saldaña-Alvarez Y, Salas-Martínez G and Orozco L: MiR-146a polymorphism is associated with asthma but not with systemic lupus erythematosus and juvenile rheumatoid arthritis in Mexican patients. Tissue Antigens 80: 317-321, 2012.

44. Lu LF, Boldin MP, Chaudhry A, Lin LL, Taganov KD, Hanada T, Yoshimura A, Baltimore D and Rudensky AY: Function of miR-146a in controlling Treg cell-mediated regulation of Th1 responses. Cell 142: 914-929, 2010

45. Tang Y, Luo X, Cui H, Ni X, Yuan M, Guo Y, Huang X, Zhou H, de Vries N, Tak PP, et al: MicroRNA-146A contributes to abnormal activation of the type I interferon pathway in human lupus by targeting the key signaling proteins. Arthritis Rheum 60: 1065-1075, 2009.

46. Kamali K, Korjan ES, Eftekhar E, Malekzadeh K and Soufi FG: The role of miR-146a on NF- $\kappa$ B expression level in human umbilical vein endothelial cells under hyperglycemic condition. Bratisl Lek Listy 117: 376-380, 2016.

47. Xie Q, Wei W, Ruan J, Ding Y, Zhuang A, Bi X, Sun H, Gu P, Wang Z and Fan X: Effects of miR-146a on the osteogenesis of adipose-derived mesenchymal stem cells and bone regeneration. Sci Rep 7: 42840, 2017.

48. Lyons JJ and Milner JD: Primary atopic disorders. J Exp Med 215: 1009-1022, 2018

This work is licensed under a Creative Commons Attribution-NonCommercial-NoDerivatives 4.0 International (CC BY-NC-ND 4.0) License. 